\title{
Comparative Evaluation of Fluoride Release From Two Different Glass Ionomer Cement and a Novel Alkasite Restorative Material - An in Vitro Study
}

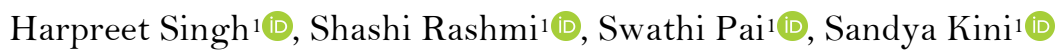

${ }^{1}$ Department of Conservative Dentistry and Endodontics, Manipal College of Dental Sciences, Manipal Academy of Higher Education, Manipal, India.

Author to whom correspondence should be addressed: Professor Dr. Shashirashmi Acharya, Department of Conservative Dentistry and Endodontics, Manipal College of Dental Sciences, Manipal Academy of Higher Education, Manipal, India. Phone: +919448121439. E-mail: sracharya@manipal.edu.

Academic Editors: Alessandro Leite Cavalcanti and Wilton Wilney Nascimento Padilha

Received: 09 September 2019 / Accepted: 14 November 2019 / Published: 22 November 2019

How to cite this article: Singh H, Rashmi S, Pai S, Kini S. Comparative evaluation of fluoride release from two different glass ionomer cement and a novel alkasite restorative material - an in vitro study. Pesqui Bras Odontopediatria Clín Integr. 2020; 20:e5209. https://doi.org/10.1590/pboci.2020.019

\begin{abstract}
Objective: To compare the fluoride release from Conventional Glass Ionomer Cement (GIC), Resin Modified GIC (RMGIC), and Cention N Alkasite Material. Material and Methods: Forty- five disc-shaped specimens of three different restorative materials (Conventional GIC, RMGIC, and Alkasite material) were made and divided into 3 groups $(\mathrm{n}=15)$. Fluoride release was evaluated at the end of Day 1, 7, 14, and Day 28 using fluoride ion-selective electrode. Intergroup and Intra-group analysis was done using One-way ANOVA with a Post-hoc test. A p-value of $<0.05$ was considered statistically significant. Results: Cention showed more fluoride release (in parts per million) than GIC and RMGIC at increased time duration. However, at the end of day 1, there was lesser fluoride release with Cention, as compared with the other groups. Conclusion: The new Alkasite restorative material showed promising results in terms of fluoride release and is better than GIC and RMGIC at increased time duration.
\end{abstract}

Keywords: Dental Materials; Glass Ionomer Cements; Fluorides. 


\section{Introduction}

Fluoride is well documented as an anti-cariogenic agent. There are several mechanisms that play their role in the anti-cariogenic capability of fluoride. Glass Ionomer Cement (GIC) is the most widely used and critically acclaimed fluoride-releasing restorative material [1]. Conventional GIC is a self-cure restorative material that has an advantage of fluoride release and biocompatibility, but its inadequate strength and toughness have limited its use in less stress-bearing areas. This limitation arose the need for newer materials with fluoride release, which includes packable GIC, compomers, giomers, and various new glass hybrid materials [2].

Resin modified glass ionomer cement (RMGIC), also called light cure glass ionomer cement was developed to overcome some of the disadvantages of conventional GIC. According to various studies, RMGIC has more flexural strength when compared to conventional GIC [3].

With time, newer and better materials for restoration have evolved, and recently "Alkasite" restorative material has been introduced [4]. Alkasite material is comparable to GIC and RMGIC because it has fluoride release and is also an aesthetic material; thus, it can be used in aesthetically concerned areas just like the latter materials. This material has relatively more translucency when compared to other glass-ionomer products, thus achieving better aesthetic properties. Moreover, it has better compressive strength than GIC and RMGIC. Therefore, Alkasite material has more diverse uses when compared to its counterparts. An extensive review of the literature yielded no documentation on the comparison of the amount of fluoride release from Alkasite material with Conventional GIC and RMGIC.

Hence this study was stemmed to evaluate and compare the amount of Flouride Release from Conventional GIC, RMGIC, and a Novel Alkasite material - Cention N at various time intervals.

\section{Material and Methods}

Materials

The restorative materials were mixed and were divided into the following groups: Group 1: Conventional GIC (GC Fuji II, GC America Corp., Alsip, IL, USA); Group 2: RMGIC (GC Fuji II LC, GC America Corp., Alsip, IL, USA); and Group 3: Alkasite material (Cention N, Ivoclar Vivadent AG, Zurich, Switzerland).

Specimen Preparation

A total of 45 disc-shaped samples from three different materials were made following the manufacturer's instructions. Conventional GIC sets through self or chemical curing. The material in its plastic state was loaded into a brass mold and pressed between two glass slabs with a transparent matrix in between. It was finally allowed to set [3]. Similar methodology was adopted for RMGIC and Cention $\mathrm{N}$ with an additional step of light curing, where the samples were light-cured from the top as well as from the bottom for 20 seconds using LED light-curing unit (Blue phase, Ivoclar Vivadent AG, Schaan, Liechtenstein) emitting blue light with intensity of $1200 \mathrm{Mw} / \mathrm{cm}^{2}$ and wavelength of $430-480 \mathrm{~nm}$ [5].

After the samples were set, they were retrieved from the mold with caution and each sample was transferred into individual polyethylene container containing $10 \mathrm{ml}$ of deionized water. [2]

Fluoride Release

The fluoride release was checked using fluoride ion-selective electrode (Orion 9609BNWP, Ion plus Sure-Flow Fluoride, Thermo Scientific, Waltham, Massachusetts, USA) coupled to a benchtop analyzer (Orion 
Star ${ }^{\mathrm{TM}}$ Series ISE Meter, Thermo Scientific, Beverly, MA, USA) with detection limit at $\pm 0.001 \mathrm{ppm}$, and the data was recorded at time intervals of 24 hours, 7 days, 14 days and 28 days. Just before the fluoride ion measurement, the stored samples were washed with $1 \mathrm{ml}$ of deionized water and then were transferred to a clean polyethylene container, containing $10 \mathrm{ml}$ of deionized water. $10 \mathrm{ml}$ of the previous solution from the plastic bottle and $1 \mathrm{ml}$ used for washing the disc was mixed with an equal amount of total ionic strength adjustment buffer. This procedure was repeated for all the samples across different time intervals. Standards containing $5 \mathrm{ppm}, 10 \mathrm{ppm}$, and $20 \mathrm{ppm}$ were used for calibration at each testing interval. The results attained were expressed as the quantity of fluoride released in parts per million (ppm).

\section{Data Analysis}

All the analysis was done using SPSS version 18. Intergroup analysis was done using One-way ANOVA with Post-hoc Tukey test, whereas for intra-group comparison one-way ANOVA with Post-hoc Bonferroni test was done. A p-value of $<0.05$ was considered statistically significant.

\section{Results}

During the inter-group analysis, Group 1 showed the highest level of fluoride release at the end of day 1, whereas group 3 showed the highest value of fluoride release at all the other time intervals. Group 3 exhibited significantly less fluoride release $(\mathrm{p}<0.001)$ at the end of day 1 when compared to Group 1 and Group 2 (Table 1).

Table 1. Inter-group analysis for fluoride release at different time intervals.

\begin{tabular}{ccccccccc}
\hline \multirow{2}{*}{ Time Interval } & \multicolumn{2}{c}{ Group 1 } & \multicolumn{2}{c}{ Group 2 } & \multicolumn{2}{c}{ Group 3 } & p-value* & Post-hoc \\
& Mean $(\mathrm{ppm})$ & SD & Mean $(\mathrm{ppm})$ & SD & Mean (ppm) & SD & & Test \\
\hline Day 1 & 23.674 & 0.922 & 20.373 & 0.782 & 5.182 & 0.526 & $<0.001$ & $1>2>3$ \\
Day 7 & 19.829 & 1.095 & 16.869 & 0.945 & 22.687 & 1.291 & $<0.001$ & $3>1>2$ \\
Day 14 & 11.465 & 0.303 & 11.633 & 0.562 & 21.481 & 0.975 & $<0.001$ & $3>1,2$ \\
Day 28 & 11.239 & 0.479 & 10.739 & 0.479 & 22.333 & 1.034 & $<0.001$ & $3>1,2$ \\
\hline
\end{tabular}

*One Way ANOVA.

Intragroup analysis (Table 2) showed the there is a significant reduction in the level of fluoride release from day 1 through day 28 . However, Group 3 shows that there is a significant rise in the fluoride level from day 1 to day 7 , and it does not increase on further days.

Table 2. Intra-group analysis at different time intervals.

\begin{tabular}{lcccc}
\hline Groups & Day & Mean & SD & p-value* \\
\hline Group 1 & 1 & 23.674 & 0.922 & $<0.001$ \\
& 7 & 19.829 & 1.095 & \\
Group 2 & 14 & 11.465 & .303 & \\
& 28 & 11.239 & 0.479 & $<0.001$ \\
& 1 & 20.373 & 0.782 & \\
Group 3 & 7 & 16.869 & 0.945 & $<0.001$ \\
& 14 & 11.633 & 0.562 & \\
& 28 & 10.739 & 0.479 & \\
& 1 & 5.182 & 0.526 & \\
& 7 & 22.687 & 1.291 & \\
*ONe Way ANOVA & 14 & 21.481 & 0.975 & \\
\end{tabular}

*One Way ANOVA. 


\section{Discussion}

This study was undertaken to evaluate and compare the amount and pattern of fluoride ion release from three different fluoride-releasing restorative materials. The various materials considered in this study are indicated for the restoration of a carious tooth in high as well as low-stress bearing areas.

The role of fluoride in exhibiting anti-cariogenic property and its remineralization potential has been discussed in the literature [6]. The filler content and nature of the glass-ionomer hydrogel matrix phase is responsible for fluoride release. The powder-liquid ratio of two-phase-systems, mixing procedure, curing time and the amount of exposed area as well as the different storage media affect the fluoride release [7]. The specimens in the current study were not coated with any adhesives or protective agents from moisture contaminants. In vitro, fluoride leached from filling materials coated with an adhesive was reduced by a factor $1.5-4[8]$.

Fluoroaluminosilicate glass is the major filler as well as the main source of fluoride release in conventional GIC and RMGIC. They have an initial high release of fluoride, which decreases progressively with time. However, Conventional GIC exhibited the highest amount of fluoride release compared to RMGIC and Alkasite material at the end of day 1. This is in accordance with the previous study [5] where, the GICs and RMGIC have high initial fluoride release ( $\geq 40 \mu \mathrm{gm} / \mathrm{cm}^{2} /$ day), but it declines rapidly with time; gradually stabilizes and plateaus within 10-20 days. This is called "burst effect" [9]

The burst effect from conventional GIC and RMGIC is probably due to the acid-base reaction. Initial superficial rinsing effect may be the cause for burst effect, whereas the plateaued fluoride release after the first day may be due to the diffusion of fluoride ion through pores and fracture lines in the cement. In general, the amount of fluoride release is directly related to the amount of fluoride already present in the cement [10].

There are several factors that influence the fluoride release from restorative materials such as storage media, temperature, and contact area of the sample to the storage media and powder/liquid ratio [11]. Fluoride release was evaluated from Conventional GIC, RMGIC, and Nano-filled GIC widely in the literature. It is observed that there was high initial fluoride release from all the tested material, which justifies the burst effect, and Conventional GIC had the maximum fluoride release, which is in partial agreement with this study $[9,12]$.

In a previous study, the estimated fluoride release from a conventional GIC, RMGIC, compomer and a resin composite, which were stored in different media including deionized water, showed that conventional GIC was the one with highest fluoride release followed by RMGIC and least was from resin composite which is in partial agreement with this study. Kinetic findings demonstrated that the conventional and resin-modified glass ionomers had a similar pattern of fluoride release; however, the amount of daily and accumulated fluoride release of these materials were different [13].

Alkasite material consists of alkaline-fillers, which produces acid-neutralizing ions. In its mixed state, the alkaline glass accounts for $24.6 \%$ by weight, which is responsible for a substantial amount of fluoride release [4]. The current study results indicate that Cention $\mathrm{N}$ had significantly low fluoride release at the end of day 1 when compared to tested conventional GIC and RMGIC. Whereas, at all the other time intervals, it has exhibited a significantly high fluoride release. This shows that Cention $\mathrm{N}$ lacks a burst effect but constantly releases fluoride over the period. Significantly high release of fluoride over the longer period may be due to a higher powder/liquid ratio and also a high amount of alkaline glass in its final state.

The capacity of remineralization of Cention N and Fuji IX was previously evaluated, and it was found that both the materials considerably reduced the demineralization of the enamel in the vicinity of the material 
when compared to non-ion releasing material. It has been hypothesized that Cention $\mathrm{N}$ may clinically inhibit caries at restoration margins [14].

Storage media influences the amount of fluoride release from the material and includes deionized water, artificial saliva, lactic acid, etc. The storage medium used in this study was deionized water. Deionized water provides near accurate readings of fluoride release because it has no ions present in it [15]. A higher amount of fluoride release has been reported in artificial saliva and the pH-cycling solution, respectively [16].

Various methods that have been employed to estimate the amount of fluoride release include spectrophotometry, ion chromatography, capillary electrophoresis, and fluoride ion electrode method. This method is equally sensitive as the standard electrode method is faster and less technique sensitive. Various authors have used this method for fluoride ion estimation [2,5,17]. The only shortcoming of using a selective ion electrode method is that it cannot detect the presence of fluoride compounds.

The release and uptake of fluoride depend not only on the fluoride content but also on the matrices, fillers, setting mechanisms, and environmental conditions of the restorative materials [18]. Limited studies are seen in the literature, which evaluates and compares the capacity of fluoride recharge in Cention.

\section{Conclusion}

The new alkasite material shows promising results on long-term fluoride release, but it lacks an initial burst effect, which limits its antibacterial property after initial placement.

\section{Authors' Contributions}

HS $\quad$ (D) 0000-0002-6998-172X $\begin{aligned} & \text { Conceptualization, Methodology, Formal Analysis, Writing - Original Draft } \\ & \text { Preparation and Writing - Review and Editing. } \\ & \text { SR }\end{aligned} \quad \begin{array}{lll}\text { Methodology, Formal Analysis, Supervision, Writing - Original Draft Preparation } \\ \text { and Writing - Review and Editing. }\end{array}$
SP $\quad \begin{array}{ll}\text { Methodology, Formal Analysis, Supervision, Writing - Original Draft Preparation } \\ \text { and Writing - Review and Editing. } \\ \text { Methodology, Formal Analysis, Writing - Original Draft Preparation and Writing } \\ \text { - Review and Editing. }\end{array}$
$\begin{array}{ll}\text { All authors declare that they contributed to critical review of intellectual content and approval of the final version to be } \\ \text { published. }\end{array}$

\section{Financial Support}

None.

\section{Conflict of Interest}

The authors declare no conflicts of interest.

\section{References}

[1] Ganss C, Lussi A. Erosive Tooth Wear: From Diagnosis to Therapy. Monographs in Oral Science. $2^{\text {nd }}$ ed. Karger Publishers: Basel; 2012. pp. 22-31.

[2] Forsten L. Fluoride release from a glass ionomer cement. Scand J Dent Res 1977; 85(6):503-4.

[3] Dionysopoulos D, Sfeikos T, Tolidis K. Fluoride release and recharging ability of new dental sealants. Eur Arch Paediatr Dent 2016; 17(1):45-51. https://doi.org/10.1007/s40368-015-0200-1

[4] Cention N. Available at: https://www.ivoclarvivadent.in/p/all/cention-n. [Accessed on 18 April, 2019].

[5] Xu X, Burgess JO. Compressive strength, fluoride release and recharge of fluoride-releasing materials. Biomaterials 2003; 24(14):2451-61. https://doi.org/10.1016/s0142-9612(02)00638-5

[6] Damen JJ, Buijs MJ, ten Cate JM. Uptake and release of fluoride by saliva-coated glass ionomer cement. Caries Res 1996; 30(6):454-7. https://doi.org/10.1159/000262359 
[7] Guida A, Hill RG, Towler MR, Eramo S. Fluoride release from model glass ionomer cements. J Mater Sci Mater Med 2002; 13(7):645-9. https://doi.org/10.1023/a:1015777406891

[8] Mazzaoui SA, Burrow MF, Tyas MJ. Fluoride release from glass ionomer cements and resin composites coated with a dentin adhesive. Dent Mater 2000; 16(3):166-71. https://doi.org/10.1016/s0109-5641(00)00003-8

[9] Upadhyay S, Rao A, Shenoy R. Comparison of the amount of fluoride release from nanofilled resin modified glass ionomer, conventional and resin modified glass ionomer cements. J Dent 2013; 10(2):134-40.

[10] Freedman R, Diefenderfer KE. Effects of daily fluoride exposures on fluoride release by glass ionomer-based restoratives. Oper Dent 2003; 28(2):178-85.

[11] Verbeeck RM, de Moor RJ, Van Even DF, Martens LC. The short-term fluoride release of a hand-mixed vs. capsulated system of a restorative glass-ionomer cement. J Dent Res 1993; 72(3):577-81. https://doi.org/10.1177/00220345930720030401

[12] Neelakantan P, John S, Anand S, Sureshbabu N, Subbarao C. Fluoride release from a new glass-ionomer cement. Oper Dent 2011; 36(1):80-5. https://doi.org/10.2341/10-219-LR

[13] Karantakis P, Helvatjoglou-Antoniades M, Theodoridou-Pahini S, Papadogiannis Y. Fluoride release from three glass ionomers, a compomer, and a composite resin in water, artificial saliva, and lactic acid. Oper Dent 2000; 25(1):20-5.

[14] Donly KJ, Liu JA. Dentin and enamel demineralization inhibition at restoration margins of Vitremer, Z 100 and Cention N. Am J Dent 2018; 31(3):166-8.

[15] Lee SY, Dong DR, Huang HM, Shih YH. Fluoride ion diffusion from a glass-ionomer cement. J Oral Rehabil 2000; 27(7):576-86. https://doi.org/10.1046/j.1365-2842.2000.00554.x

[16] Carvalho AS, Cury JA. Fluoride release from some dental materials in different solutions. Oper Dent 1999; 24(1):14-9.

[17] Shiozawa M, Takahashi H2, Iwasaki N. Fluoride release and mechanical properties after 1-year water storage of recent restorative glass ionomer cements. Clin Oral Investig 2014; 18(4):1053-60. https://doi.org/10.1007/s00784-013-1074-4

[18] Wiegand A, Buchalla W, Attin T. Review on fluoride-releasing restorative materials - fluoride release and uptake characteristics, antibacterial activity and influence on caries formation. Dent Mater 2007; 23(3):343-62. https://doi.org/10.1016/j.dental.2006.01.022 